\title{
Corollarium Technologies
}

\author{
Bruno Barberi Gnecco \\ Corollarium Technologies Ltd. - São Paulo - Brazil \\ https://corollarium.com \\ brunobg@corollarium.com
}

\begin{abstract}
Corollarium is a company that specializes in web and mobile development, image processing, virtual and augmented reality. Its expertise encompasses the full development process - from design to production to installation, aiming to offer customized solutions of the highest quality and lowest maintenance. This paper goes briefly over what we have been researching and creating over the last years.
\end{abstract}

Keywords: virtual reality; augmented reality; image processing; simulators.

\section{Introduction}

Focused on technology for data visualization, Corollarium is a company that specializes in image processing, virtual and augmented reality, with desktop, web and mobile development. Its expertise encompasses the full development process-from design to production to installation, aiming to offer customized solutions of the highest quality and lowest maintenance. Corollarium has partnerships with international academic institutions and develops state-of-the-art research and technology. It has also developed projects and sold software licenses in Brazil, Europe, Asia and the Americas.

The company has a long history of its projects being approved for relevant and well recognized grants. It was approved for a FAPESP PIPE grant in 2009, with a low cost immersive projection system project. In 2010 it was approved in the PRIME project of FINEP, as well as the XPTA.lab project of the Ministry of Culture, in partnership with UFSCar, UNESP, UFPA, UFPB and Abútua Tecnologia. In 2011, it received a RHAE grant from CNPq that lasted until 2013, and was renewed for a second phase from 2015 until 2017. It was chosen to be part of the Brazilian delegation at the Museum Connections world fair, which took place in Paris in 2016.

Corollarium has always researched and developed with universities. Its partnerships over the last few years have included UFSCar, UNESP, UNICAMP, UFSJ and UNIFESP, with labICE [1]. We believe that these partnerships benefit both the company and the university, exchanging experience, technology and helping to bridge the gap between the commercial market and academic research.

Over the years our projects have often handled problems of real-time synchronization, on image acquisition, data processing and display. These are challenging problems, dealing with all usual issues from distributed processing, and facing the difficulties of running in unexpected venues where there is little control of the environment. We have learned to design and implement robust solutions, understanding that customers want not only a plug-and-play solution, but a zero-config, zero-maintenance that just works, even with sketchy networks, faulty internet connections and unusual hardware. As a result our products have evolved and run on many operating systems (Linux, Windows,
OSX, Android, iOS) and processor architectures (ARM, x86) and networks (WiFi, Ethernet, 4G).

\section{Main Projects}

Ever since it was created, Corollarium designed and developed expert software for a multitude of customers. Our expertise in XR led us to develop technology for multi-projection systems, including the design, project and installation of mini CAVEs with commodity and low cost devices. Our knowledge allows us to perform the entire flow of a VR system implementation, both hardware and software.

Being hired by customers to develop projects has always been a mainstay of the company. These projects fall under non-disclosure agreements and therefore cannot be exposed here. But the research and development made through the years led us to create a considerable amount of technology, which besides allowing us to be faster and more efficient, it also enabled us to develop our own products, some of which are described below.

\subsection{Camera360}

Camera360 is a multi-camera system that takes photos in synchrony [2]. With one click you have images from every angle and all details. Camera360 captures an image around an object with multiple cameras. The photos are captured and processed in seconds and immediately available in the cloud, on a site with its own domain, using the customer's logo and visual identity, ready to be shared on social networks. We have used Camera360 for photogrammetry as well, generating $3 \mathrm{D}$ content from pictures and using it for augmented and mixed reality. It is a cost-effective system that uses smartphone cameras or modular computers, improving the traditional technique of using high-cost cameras and complex electronics for synchronization. Camera360 systems of up to 80 cameras have been built.

Camera360 can be used for 360 degree photos, panoramas, bullet time, promotional events, interactive catalogs, fashion, art, 3D reconstruction and more. Figures 1 and 2 show real deployments of the system. 


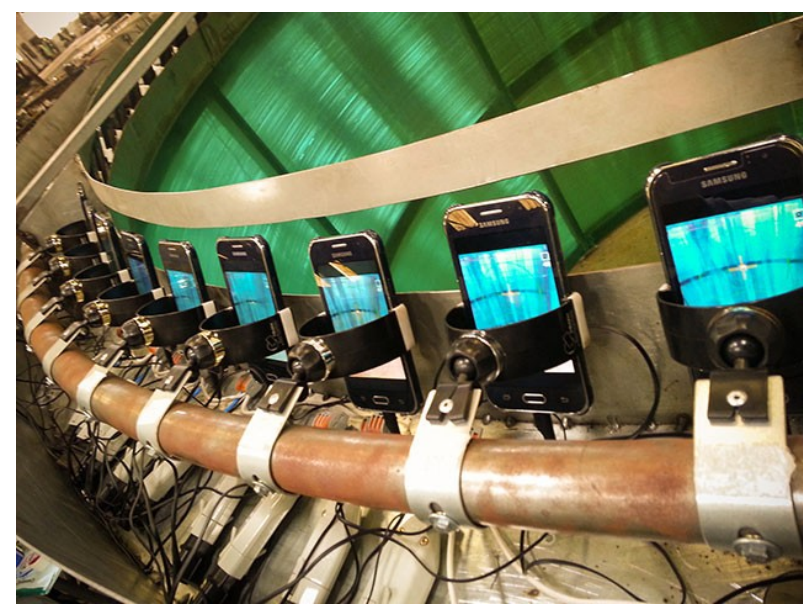

Figure 1. A Camera360 installation using Android.

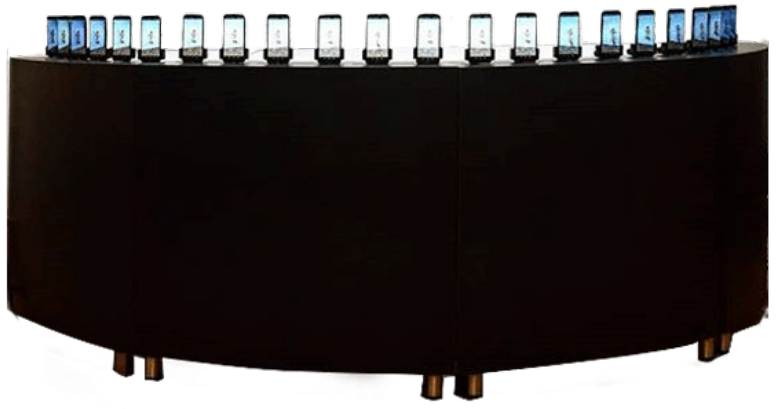

Figure 2. Another installation of Camera360 made by a customer.

Synchronization of cameras has first been done by Edward Mulbridge, and the bullet-time effect became famous through the Matrix movies. It entails high speed synchronization and capture of images, usually through special hardware. We have evolved our solution from point-and-shoot cameras to Android and Raspberry Pi devices, understanding the void in the market for low and mid-range solutions. The lack of a dedicated electronic trigger, which has near zero latency, is a challenge. Wifi networks have considerable jitter, delays and lack of multicasting, and even with Ethernet one has to take into account a number of delays: the network layers, operating system, camera shutter etc.

We have also taken great care of the usability: a Camera360 installation at a large venue, such as our installation in the Coachella festival in California, generates a line of people interested in taking pictures. We needed a fast, reliable system that took pictures from many cameras, gathered them into a single server, processed them for alignment, color correction, watermarking, generated GIFs and videos and uploaded them to the cloud with a single click.

\subsection{Multiselfie}

Multiselfie is a mobile and web app to take photos with multiple devices at the exact same time [3]. Only one person clicks and all the phones take pictures together. It's a consumer version of Camera360.
With Multiselfie phones from several people are used to take pictures at the exact same time. Everyone gets their phone out and gets ready, and a single person creates a room, just like creating a chat room. They get a link that is shared among everybody, and only the person that created the room needs to click a button to shoot a picture. You have multiple photos of a single moment, and instead of a logistic problem you have fun taking the picture. Figure 3 shows three phones running Multiselfie.

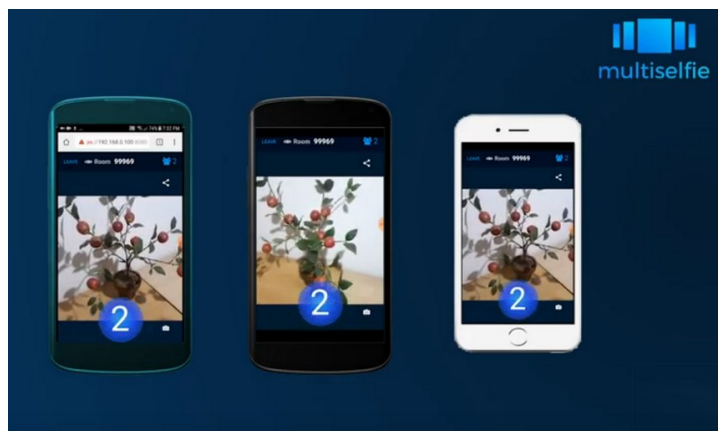

Figure 3. Multiselfie over three different phones.

In larger events we can integrate Multiselfie into other apps or sites, and give a more fine grained control to the responsible person, as well as information about the pictures and analytics. Multiselfie added new challenges to an already difficult problem. How to synchronize several - perhaps dozens, perhaps hundreds - phones over different 3G, 4G and Wifi networks? How to deal with the ensuing upload flood? We developed special synchronization algorithms that wouldn't need an immediate trigger signal, which is unfeasible in these circumstances. Edge computing came to the rescue, processing as much as possible on the phones before uploading data to the cloud.

\subsection{Video wall}

Our video wall controller software is an inexpensive and robust video wall solution with a friendly web interface and an excellent cost/benefit ratio [4]. It handles multiple screens with all kinds of content: videos, images, camera, window and desktop streaming.

We support clients in Windows, Linux and Android, including x86 and ARM architectures. Using Raspberry Pis as zero-clients makes our solution one of the most cost-effective in the market, with trivial deployment.

The visual design mode, as shown in figure 4, enables any transformation on the screens: they can be arbitrarily spaced and rotated, with real time updates. The software automatically applies the transformations, and even traditional matricial arrangements are benefited by the fine screen positioning, compensating for borders, as shown in figure 5. There's a very fine video synchronization algorithm that compensates for differences in timing with a smooth speed scaling function, avoiding sudden frame jumps. 


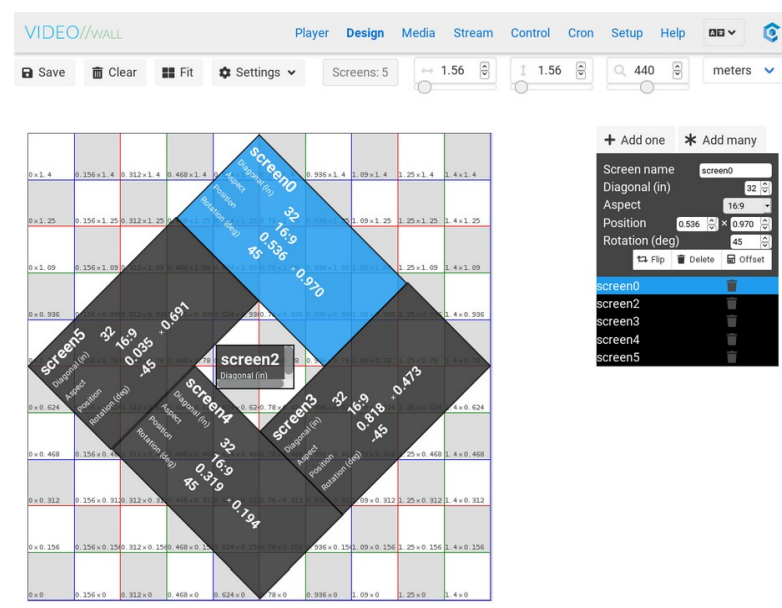

Figure 4. The design system for videowall screens.

We provide several features useful for both marketing applications and operation centers. These include scheduling of operations (such as turning the screens on and off), streamcasting of applications and the whole desktop of other machines with zero software installation, displaying messages and more.

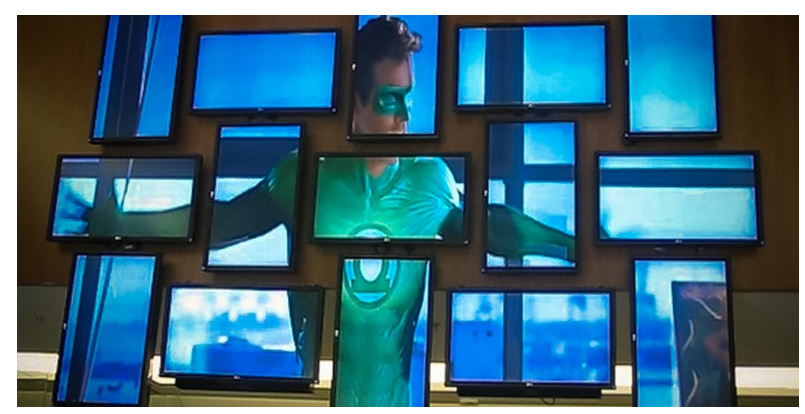

Figure 5. One of our deployed video walls.

\subsection{Virtuactor}

Virtuactor is an interactive 3D simulator application for industrial, commercial and risky operations.

It is a completely customizable solution for training and evaluating people using a simulated computer environment. Its goal is both to provide rapid, risk-free initial training and ongoing user assessment, to ensure that processes are being followed and to provide tools for optimizing bottlenecks.

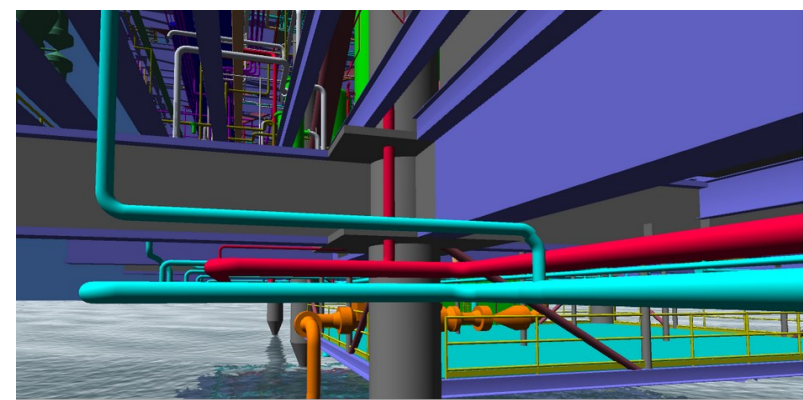

Figure 6. Virtuactor simulating an oil platform.
Risky operations, production training, optimization and evaluation of people - Virtuactor allows you to simulate common or emergency situations and complex and delicate operations, as is the case of oil platforms (figure 6). The results reports are generated by a simple web interface for human analysis, with automatic evaluation of performance through artificial intelligence. Simulations save time and money and also avoids damages that could occur in equipment due to irregular operation. Virtuactor can be used on screens, with virtual reality glasses or even in immersive virtual reality environments.

\subsection{LajeVR}

This project uses virtual reality to simulate a dive on a 3D model of the Laje de Santos State Marine Park [5]. It includes the island above water and the submerged area, allowing people from all over Brazil and the world to visit the park virtually, do simulated virtual dives, learn about the fauna that inhabits or migrates through the park and the marine environment.

The Laje de Santos State Marine Park (PEMLS) is the first Marine Park among the Conservation Units of the State of São Paulo, created on September 27, 1993, through State Decree $n^{\circ}$ 37.537. It has the objective of protecting marine biodiversity, and conserving biological diversity on the coast of the state of São Paulo. It is located about $40 \mathrm{~km}$ from the coast of the city of Santos.

As there are no other rock formations or islands nearby, it has a large concentration of fish in passing and reefs, being one of the main diving and underwater photography spots in the country. The Park also has a wreck. It can be visited by boats, but landing on the island is forbidden. The navigation is long, about $1 \mathrm{~h} 30$ to $2 \mathrm{~h}$ in speedboats or $6 \mathrm{~h}$ to $8 \mathrm{~h}$ in slow boats, which makes the visit difficult and of considerable cost, being basically restricted to accredited scuba divers. With that few people have access to a place of enormous beauty and diversity of fauna.

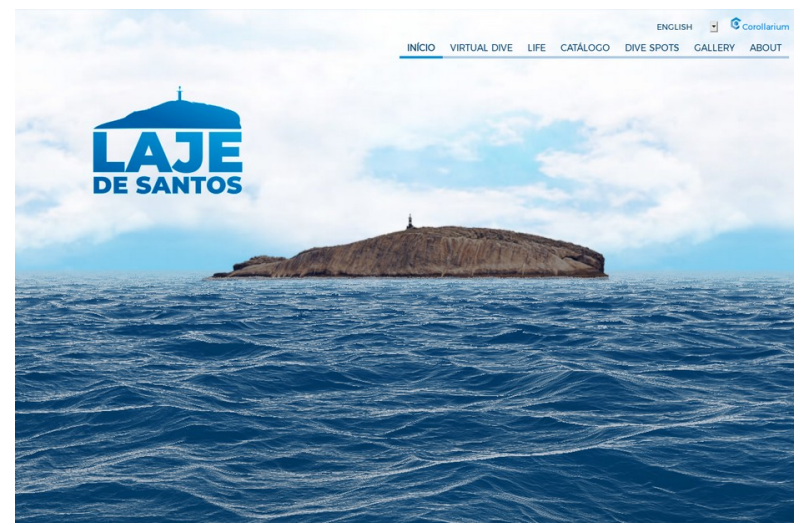

Figure 7. Laje de Santos in our 3D simulation.

Since we aimed for a large audience, the project is available at a public website and we used WebGL for the $3 \mathrm{D}$ and VR parts (figure 7). The project is sponsored 
by a Proac grant from the State of São Paulo. It is an ongoing project, delayed due by the COVID pandemic. The entire project is being released as open source, and the current version can be visited at https://corollarium.github.io/lajevr/.

\section{Social, technological and/or scientific contributions}

Most of our internal research has been in better technology for distributed computing, particularly concerning synchronization under different circumstances. Our technology for simultaneous image processing has been used for several applications, including entertainment, marketing, culture and industry.

We have also created a considerable amount of technology on image and video processing, which has been applied on several projects for our customers. An automatic alignment and color calibration software is integrated with Camera360. Other applications include automatic recognition of patterns and entities, often using machine learning.

Our usage of commodity hardware for VR environments results in installations that cost five to ten times less than high end solutions available on the market, making it affordable to universities and companies that would not be able to have it otherwise.

Our continuous collaboration with universities led to a two-way transference of technology, both bringing academic research to the market and helping academic projects to be implemented.

On the social side, we are particularly proud of the LajeVR project, since we can take people to see a marine park which is not easily accessible. We can take millions of people to see a preserved underwater area which is seen by only a few hundred people each year, and for free. We have often collaborated with open source projects, writing bug fixes and improvements. We have also released our own, most of which are available at our Github page [6].

\section{References}

[1] Guimarães, M.P.; Dias, D. R. C.; Mota, J. H.; Gnecco, B. B.; Durelli, V. H. S.; Trevelin, L. C. (2018) Immersive and interactive virtual reality applications based on 3D web browsers. Multimedia Tools and Applications 77(1): 347361. DOI: $10.1007 / \mathrm{s} 11042-016-4256-7$.

[2] Camera360 website. https://camera360.com.br Accessed on August $10^{\text {th }}, 2020$.

[3] Multiselfie. https://multiselfie.com Accessed on August $10^{\text {th }}, 2020$.

[4] Corollarium Videowall website. https://softwarevideowall.com Accessed on August $10^{\text {th }}$, 2020.

[5] LajeVR project. https://corollarium.github.io/lajevr/ Accessed on August $10^{\text {th }}, 2020$.

[6] Corollarium Github Page. https://github.com/Corollarium/ Accessed on August 10 2020. 